\title{
INFECTIOUS DISEASES.
}

UNDER THE CHARGE OF

CLAUDE B. KER, MI.D.

\section{Inhalations of Hot Air in the Treatment of Diphtheria.}

Rendu (Lyon Médical, 12th January 1913) reports a series of cases of diphtheria in which the throat lesions were treated by heated air, applied by means of a tube. In his view such inhalations cause an immediate relief to the sore throat, exercising a sort of analgesic action. The hyperæmia which follows may also be of value, as may the increase in glandular secretion which appears to be excited. But the principal action he regards as a bactericidal one, and he suggests that, if the bacilli are not actually destroyed, their virulence and power of producing toxin is very much diminished. Rendu disclaims any idea of substituting this method of treatment for the use of serum, but considers that the two methods can very well be carried out together. Nevertheless to test the value of the hot air he treated two series of cases, one, as usual, with serum, and the other by his new method, and found very little difference in the results. He tabulates in his paper the main features of the cases composing each series, and points out that, so far as severity of type is concerned, there is little to choose between the two groups. 'The air, heated in a contrivance analogous to the electric drying apparatus of hairdressers, was administered through a flexible metallic tube, the nozzle passed into the mouth and suitably protected. The temperature attained ranges up to $110^{\circ} \mathrm{C}$. The heated air may be given as long as tolerated, and then intermitted for a minute or two, and a séance lasts about 5 minutes. The average patient had 4 or 5 séances in the first three or four days. The hot air appeared to cause the disappearance of membrane at least as rapidly as did serum. The fever on an average was only slightly more prolonged. One patient, however, admitted free from laryngeal symptoms, developed croup subsequent to the commencement of hotair treatment, while, as we would have confidently predicted, no such accident occurred in the serum-treated group. With this exception there is very little difference in the results of the two series of cases, each of which included 33 patients, the serum series having only a slight advantage in the case mortality $-24 \cdot 24$ per cent. as against $27 \cdot 27$ per cent. The deaths, however, appear in many instances to have been due to broncho-pneumonia and not to uncomplicated diphtheria; there were no cardiac deaths for instance, and the palate was the only situation in which paralysis manifested itself. Many of the patients, moreover, appear to have contracted measles, and this in reality was the cause of death. One is tempted to conclude that if a similar experiment was repeated in cases of uncomplicated diphtheria 
of a somewhat more severe type, the patients treated by hot air alone would not compare so favourably with serum-treated cases. It is also a little difficult to see how a temperature great enough to destroy or limit the activities of the bacilli could be tolerated sufficiently long by the patient. Rendu tells us that children of from 4 to 8 years in a séance of 5 minutes inhale air at from $20^{\circ}$ to $60^{\circ} \mathrm{C}$. for 2 minutes, $70^{\circ}$ for 20 seconds, $90^{\circ}$ for 15 seconds, $100^{\circ}$ for 10 seconds, and $110^{\circ}$ for 5 seconds, older children being of course more tolerant. This does not seem to expose the germs in the membrane to a high temperature for long enough to modify them effectively. Rendu's paper is none the less very interesting, although we hardly think he will find many imitators. He suggests that, as a substitute for serum treatment, his method might fairly be indicated in tubercular subjects, in patients who have previously had a dose of serum and in whom anaphylaxis is dreaded, and in patients who have just had another infectious disease such as measles, as in the last-named circumstances serum is often very disappointing.

\section{Treatment of Erysipelas by Vaccines.}

Yoshihiro Takaki (Sei-i-kwai Med. Journ.) has treated 16 cases of erysipelas by vaccines. In some of the cases a stock streptococcus vaccine was used, but in the majority of the patients the vaccine employed was autogenous. Although many of the cases were very serious, in no instance did death result. When the condition came early under treatment the illness was, in Takaki's opinion, shortened, and in certain cases the effect was almost instantaneous. Auto-vaccines were found more efficacious than stock vaccines. The exact dosage is not given, but injections appear to have been made at intervals of two days. A study of the cases in detail suggests that the stock vaccine was of little, if any, value. It is true that the inflammatory process was apparently checked abruptly in some cases, but erysipelas is a disease of such uncertain duration, and so often terminates by crisis, even when no specific treatment is used, that we are not very impressed with the results. On the other hand the use of the auto-vaccine was somewhat more successful. Here again, however, if we apply the test of the number of recurrences, the treatment does not seem convincing. Three patients suffered from relapse-a high proportion even for untreated cases. On the whole, therefore, granting that a number of severe cases did extremely well, it would be unsafe to lay very much stress on the value of a method of treatment which is still, after all, only in the experimental stage.

\section{Serum Treatment of Whooping-Cough.}

Rosenthal (Arch. gén. de méd., January 1913) gives an interesting summary of the recent work of the Russian observer Klimenko on 
whooping-cough. The bacillus of Bordet and Gengou is not yet entirely accepted as the cause of the disease. The chief evidence in its favour is the fact that it is in many cases agglutinated by the serum of convalescents, and that the latter gives constant positive reactions with the deviation of complement test. Klimenko has carried the researches of the French writers to a further stage. While he failed to produce whooping-cough in most laboratory animals, he was successful in causing an analogous condition in puppies and monkeys by means of inoculation of the nasopharynx with the bacillus in question. The cough set up, however, was not spasmodic, although sometimes followed by vomiting. Puppies often died of pneumonia some weeks after inoculation, and the inflamed areas of lung gave cultivations of the Bordet-Gengou bacillus. Klimenko failed in his endexvours to procure an active toxin, and concludes with the French observers that their bacillus only elaborates an endotoxin. This naturally made it unlikely that an active therapeutic serum could be prepared. Immunising experiments, however; were carried out on rabbits, dogs, goats, and sheep. Intramuscular or subcutaneous injections always caused necrosis of tissue locally, and intravenous injections were ultimately employed. Forty-eight-hour-old cultures on gelose in sterile saline solution were used, at first previously killed by heat, and later in the living state. Many of the animals, however, died in the process of attempted immunisation. The serum, nevertheless, when obtained was proved experimentally to have considerable power. Injected into laboratory animals previous to the inoculation of 2 or 3 lethal doses, it prevented the fatal result. It possesses agglutinating power ( 1 in 5000), contains specific opsonins, and produces deviation of complement. Klimenko also treated 35 patients with the preparation, using hypodermic doses of from 25 to 50 c.c., or rectal doses of from 50 to 100 c.c. He considers that while his serum does not abort the course of whooping-cough, it invariably diminishes the violence of the accesses, and also the number of whoops. Curiously enough the effect on the cough was most noticeable at night. The duration of the illness appeared to be shortened, but the figures given are not very convincing. It would be very gratifying to find a really scientific and successful treatment for this distressing disease, but the difficulties in the production of serum seem so great, and the action so uncertain, that it does not appear that in the meantime we can expect much from the method.

\section{A Municipal Tuberculosis Sanatorium.}

The Boston Medical and Surgical Journal (23rd January 1913) contains an interesting review of a report of Dr. H. M. Biggs of the New York City Health Department on the sanatorium at Otisville. This institution appears to be constructed entirely on the "outdoor" 
principle, that is to say, of 500 patients not more than 25 can under any possibility be placed within 4 walls. This is the more remarkable when it is understood that many second- and third-stage patients are treated, and that the atmospheric temperature may fall to zero in winter. The grounds are very extensive-between 1300 and 1400 acres. Originally it was intended that the institution, like other sanatoria, should care only for cases of incipient disease, but it is stated that experience has shown that there were many reasons for changing this view. Perhaps the most important is that in the majority of cases occurring among the poor the disease, when recognised, is no longer incipient. Incipient cases, moreover, are so little troubled by symptoms that many individuals will not stop work and enter it sanatorium. On the other hand, cases in the second stage of the disease do often quite as well as the incipient ones, and even welladvanced third-stage cases often show a surprising improvement. Advanced cases are therefore taken at Otisville, and the idea is, if they do not show improvement after two or three months, to transfer them to hospitals for advanced cases in the city. The New York scheme, then, is remarkably comprehensive, and the patients at Otisville illustrate all the stages of tuberculosis. The recognition, in this manner, of the claims of the second-stage case is satisfactory, as too often in Edinburgh such cases have, as it were, fallen between the two stools of the sanatorium and the hospital for advanced cases, being too advanced for the first and not sufficiently ill for the second. The Insurance Act, however, is removing this anomaly.

Typhus Fever in Boston.-The curious sporadic cases of fever, described by Brill of New York as occurring not infrequently in that city, have, as most of us who were interested in the subject confidently expected, been definitely identified with typhus fever. Lee (Boston Med. and Surg. Journ., 23rd January 1913) has subjected to a critical review the medical records of the Massachusetts General Hospital to ascertain whether Boston as well as New York unconsciously harboured this particular infection. The cases investigated were those clinically classed as typhoid fever, and all cases of indefinite fever during the last 10 years. I.ee succeeded in finding records of 28 cases which corresponded fairly well to Brill's description of the disease, which was for some years called by his name, and is now generally regarded as mild typhus fever. These cases gave negative blood cultures and negative Widal reactions to both the typhoid and paratyphoid bacillus. They were scattered fairly evenly through the period investigated, but 17 of them occurred in the four months, June to September. In 15 there was a definite crisis, and the lysis in the remainder did not take more than 72 hours. It is interesting to note that, as was the case with the patients treated by Brill, a very high proportion came from Eastern Europe, 18 of the 28 having been born in Russia, and only 
3 in the United States. The most important feature of the recent observations of American physicians on this disease is that typhus fever must now be regarded in a new light, and that it is capable of a sporadic existence, and may show an extremely low degree of infectivity and also a very trivial death-rate. It is curious that these modified cases have not yet been identified in Great Britain, where small outbreaks of the disease still occur.

\section{MENTAL DISEASES.}

UNDER THE CHARGE OF

JAS. MIDDLEMASS, M.D., ANd DOUGLAS M־RAE, M.D.

\section{Insanity Due to Tumour of Thyrold.}

In the Gazette des Hôpitaux (5th November 1912, p. 1745) Drs. Horand, Puillet, and Morel relate a case of insanity due apparently to a tumour of the thyroid. The patient was a man aged 39, who, from the age of 9 , suffered from a swelling in the neck. It grew in size very slowly until about a year before he was admitted to hospital, when the tumour had become so large that he decided to have it removed. For many years he was looked on as an eccentric. He wanted to know everything, and read many books, chiefly of a scientific kind. He had formed his own ideas of Creation, of Nature, and of many other matters, and had formulated them in a system which appeared to him perfectly satisfactory. He had embodied these in a voluminous treatise, which, however, no one would publish, so in a fit of pique he burned it. He also suffered from hallucinations of many kinds. $\mathrm{He}$ imagined himself to be animated by a spirit which he named Elébram, and which inspired all his thoughts. His intelligence seemed latterly somewhat degraded from its previous level, which admittedly was above the average. The tumour was completely removed and found to be a mass of caseous material, such as is found in some dermoid cysts. The wound healed rapidly and satisfactorily. There was subsequent to the operation a remarkable mental change. After his return home he was reported by his relations to have resumed a normal life, all mental symptoms having disappeared. It is, of course, not an unusual thing for an insane person to recover after an operation of any kind, but the facts of this case seem to strengthen the view that the thyroid has a specially intimate relation to brain activity.

\section{Mental Disturbances Produced by Thyroid Feeding.}

As thyroid medication has been strongly advocated as a remedy in certain cases of insanity, and is extensively used for this purpose, it is perhaps as well that attention should be drawn to some of the risks that may be incurred in so doing. Professor Roque describes (Progrès méd., 20th July 1912, p. 354) a case in which acute mental symptoms 